\title{
IMPACTO EN LOS DESEMPEÑOS DIARIOS ORIGINADOS POR CARIES DENTAL EN ESCOLARES DE 6 AÑOS EN LA PARROQUIA CAÑARIBAMBA - CUENCA, 2016
}

\section{IMPACT ON THE ORIGINAL DAILY DENTAL CARIES PERFORMANCES IN 6 YEAR - OLD SCHOOLCHILDREN AT CAÑARIBAMBA - CUENCA, 2016}

\author{
Oyervide-Soto Catalina. ${ }^{1}$ *, Sarmiento-Criollo Patricio. ${ }^{2}$ Paladines-Calle Stephanie. ${ }^{3}$ \\ ${ }^{1}$ Odontóloga Rural del Ministerio de Salud Pública del Ecuador. Ecuador. \\ ${ }^{2}$ Catedrático de la Carrera de Odontología de la Universidad Católica de Cuenca. Ecuador. \\ ${ }^{3}$ Estudiante de la Carrera de Odontología Universidad Católica de Cuenca.Ecuador. \\ *cataoyervide-27@outlook.com
}

\begin{abstract}
Resumen
Objetivo: Relacionar la caries dental y el impacto que genera en los desempeños diarios en los escolares de 6 años de edad de la parroquia Cañaribamba del cantón Cuenca, provincia del Azuay en el año 2016. Materiales y Métodos: Se realizó un estudio retrospectivo y cuantitativo en 96 escolares, mediante la recolección de datos en el programa EPI (Índice ceod, ChilOIDP), en el cual se registró los datos de las fichas epidemiológicas de la base de datos de la Universidad Católica de Cuenca. Resultados: Mediante el índice ceod se determinó el total general de dientes cariados, extraídos y obturados, dando como resultado un total del $54 \%$ en el género masculino mientras que en el género femenino el total fue del $46 \%$, representando un promedio general de ceod 5,47 (nivel de severidad alto). Por otra parte el $67 \%$ del total de la muestra generó impacto en su desempeño diario por la presencia de caries, y el $33 \%$ no presentó impacto a pesar de la presencia de la misma; el desempeño con mayor impacto fue "comer alimentos" con $16 \%$ en los hombres y "contactarse con otras personas" con un $15 \%$ en las mujeres. Conclusiones: Al analizar el índice ceod y el impacto en los desempeños diarios según el sexo, se obtuvo mayor porcentaje en el grupo masculino; recalcando que el total de la muestra de estudio no es cuantitativamente equivalente entre ambos géneros; teniendo una relación de 1.5:1.
\end{abstract}

Palabras clave: Caries dental, calidad de vida, impacto, ceod.

\section{Abstract}

Objective: Relate dental caries and the impact it generates on daily performances in 6-year-old schoolchildren from the Cañaribamba parish of Cuenca, province of Azuay, in the year 2016. Materials and Methods: A retrospective and quantitative study In 96 schoolchildren, through the collection of data in the EPI program (Index ceod, Child-OIDP), in which the data of the epidemiological records of the Catholic University of Cuenca database were recorded. Results: The general total of decayed, extracted and obturated teeth was determined using the ceod index, resulting in a total of 54\% in the male gender, while in the female gender the total was 46\%, representing a general average of ceod 5.47 (high severity level). On the other hand, $67 \%$ of the total sample had an impact on their daily performance due to the presence of caries, and $33 \%$ had no impact despite the presence of caries; the performance with the greatest impact was "eating food" with 16\% in men and "contacting other people" with 15\% in women. Conclusions: When analyzing the ceod index and the impact on daily performance according to sex, a higher percentage was obtained in the male group; emphasizing that the total of the study sample is not quantitatively equivalent between both genders; having a ratio of 1.5: 1 .

Key words: Malocclusion, child, prevalence.

\section{INTRODUCCIÓN}

La caries dental se ha convertido en un problema de salud bucal a nivel mundial, presentándose como una patolo- gía dinámica y crónica que se presenta por múltiples causas como: biológicas, sociales, económicas, culturales y ambientales. ${ }^{1-3}$ La caries se exterioriza en la estructura dentaria al 
entrar en contacto con los depósitos microbianos y por el desequilibrio entre la sustancia dental y el fluido de la placa circundante, cuyo signo es la destrucción localizada de los tejidos duros. Su formación y desarrollo están condicionados por el modo y estilo de vida. ${ }^{4-6}$ La calidad de vida relacionada con la salud, abarca aspectos tanto para el funcionamiento físico, mental y el bienestar de las personas. Para evaluar el impacto en los desempeños de la salud bucal, se analizaron 8 indicadores pertenecientes al Test Child-OIDP, los cuales son: comer alimentos, hablar claramente, lavarse los dientes, descansar, mantener buen estado de ánimo, sonreír, contactarse con otras personas. ${ }^{7-9}$ Según la organización mundial de la salud (OMS), el $99 \%$ de la población padece de caries. ${ }^{10,11}$ En la rama de la odontología, se establecen tres niveles con la finalidad de medir la calidad de vida relacionada con la salud: el primero, refiere a la situación de la salud bucal, el segundo, referido a los primeros impactos negativos causados por las condiciones bucales (dolor, incomodidad, limitación funcional e insatisfacción con la apariencia), el tercero, aquellos que afecta el desarrollo de las actividades diarias para las cuales se requieren una adecuado rendimiento físico, psicológico y social. ${ }^{12-14}$ Actualmente la odontología presenta variadas y diversas áreas, siendo la cariología una de las más relevantes al evaluar la relación existente entre la caries y el impacto en los desempeños diarios en escolares de 6 años, por tal motivo se realizó este estudio en la parroquia Cañaribamba del cantón Cuenca en el año 2016, el cual nos permite observar la prevalencia de caries y su impacto en los distintos desempeños diarios.

\section{MATERIALES Y MÉTODOS}

La población del estudio fue de 96 fichas epidemiológicas de la parroquia Cañaribamba, lo cual corresponde al total de fichas registradas en el estudio epidemiológico de salud bucal las cuales reposan en el centro de Investigación de Odontología en el mapa epidemiológico de la Universidad Católica de Cuenca. Se incluyeron en el presente estudio las fichas epidemiológicas con el respectivo consentimiento informado, firmado por parte de los representantes de los escolares, matriculados en los centros educativos de la parroquia Cañaribamba en el cantón Cuenca, en el periodo 2015 - 2016. Para la recolección de datos, se utilizó una ficha de información digital en el programa de libre acceso EPI INFO, en el cual se han registrado los datos que constan en las fichas epidemiológicas de la Universidad Católica de Cuenca, las cuales presentan 5 partes, la primera datos generales de los pacientes, la segunda; de datos de índice de higiene oral, la tercera de índice de caries ceod, la cuarta de índice de enfermedad periodontal de Russel y la quinta de procesador Core 5. Para llevar a cabo el estudio se necesitaron: recursos institucionales (UCACUE, ZONAL 6 de Educación), recursos humanos (Examinadores y tutores) y recursos financieros (autofinanciados). La investigación se realizó entre los meses de septiembre y octubre de año 2016, recolectando datos de fichas epidemiológicas que reflejan la situación epidemiológica del año en curso, dichas fichas fueron tomadas desde el mes de Mayo a Julio del 2016. El estudio de caries dental y la relación que tiene con el impacto diario buscó describir cuantitativamente y cualitativamente el problema en escolares de 6 años de edad, utilizando los parámetros de la OMS, para diagnosticar presencia de caries dental.

\section{RESULTADOS}

El presente estudio fue realizado con los datos epidemiológicos obtenidos de los escolares de la parroquia $\mathrm{Ca}$ ñaribamba, que constan en el archivo del departamento de investigación de la carrera de odontología de la UCACUE en el periodo 2016, mostrando los siguientes resultados: En lo referente a la distribución de la muestra de los escolares de 6 años de la parroquia Cañaribamba-Cuenca en el 2016 de acuerdo al sexo se pudo observar que existió un mayor porcentaje de escolares de sexo masculino $(58 \%)$ en relación al sexo femenino (42\%). (Tabla 1 )

\begin{tabular}{lll}
\hline & $\mathbf{n}$ & $\%$ \\
\hline FEMENINO & 40 & $42 \%$ \\
MASCULINO & 56 & $58 \%$ \\
Total general & 96 & $100 \%$ \\
\hline
\end{tabular}

Tabla 1. Distribución de la muestra de acuerdo al sexo

Prevalencia de caries en escolares de 6 años en la parroquia Cañaribamba-Cuenca 2016 de acuerdo al sexo. (Tabla 2) El sexo masculino presento mayor porcentaje de prevalencia de caries $(46 \%)$ en relación al sexo femenino (39\%).

\begin{tabular}{lcccccc}
\hline & \multicolumn{2}{c}{ FEMENINO } & \multicolumn{2}{c}{ MASCULINO } & \multicolumn{2}{c}{ Total general } \\
\hline & $\mathrm{n}$ & $\%$ & $\mathrm{n}$ & $\%$ & $\mathrm{n}$ & $\%$ \\
ENFERMO & 37 & $39 \%$ & 44 & $46 \%$ & 81 & $84 \%$ \\
SANO & 3 & $3 \%$ & 12 & $13 \%$ & 15 & $16 \%$ \\
Total general & 40 & $42 \%$ & 56 & $58 \%$ & 96 & $100 \%$ \\
\hline
\end{tabular}

Tabla 2. Prevalencia de caries de acuerdo al sexo.

En cuanto a la Relación de la caries y su impacto en los desempeños diarios en escolares de 6 años de la parroquia Cañaribamba-Cuenca 2016 (Tabla 3), el $67 \%$ presentó impacto en los desempeños diarios a causa de esta patología, mientras que el $33 \%$ no presentó impacto alguno.

Según el sexo, el impacto de la caries con respecto al desempeño diario en escolares de 6 años de la parroquia Cañaribamba- Cuenca 2016 (Tabla 4), se observó que el desempeño con mayor impacto en el sexo femenino fue "contactarse con otras personas" presentando un efecto leve según la severidad, en cuanto al grupo masculino el desempeño con mayor impacto fue "comer alimentos" presentando un efecto leve según la severidad. 


\begin{tabular}{|c|c|c|c|c|c|c|c|c|c|c|c|c|c|c|c|c|}
\hline \multirow[b]{2}{*}{ Sexo } & \multicolumn{2}{|c|}{ Comer } & \multicolumn{2}{|c|}{ Lavarse los } & \multicolumn{2}{|c|}{ Hablar } & \multicolumn{2}{|c|}{ Descansar } & \multicolumn{2}{|c|}{ Ánimo } & \multicolumn{2}{|c|}{ Sonreir } & \multicolumn{2}{|c|}{ Labores } & \multicolumn{2}{|c|}{ Contactarse } \\
\hline & No & Si & No & $\mathrm{Si}$ & No & $\mathrm{Si}$ & No & Si & No & $\mathrm{Si}$ & No & $\mathrm{Si}$ & No & $\mathrm{Si}$ & No & $\mathrm{Si}$ \\
\hline Femenino & \multicolumn{2}{|c|}{$67,5 \% 32,5 \%$} & \multicolumn{2}{|c|}{$70,0 \% 30,0 \%$} & \multicolumn{2}{|c|}{$67,5 \% 32,5 \%$} & \multicolumn{2}{|c|}{$80,0 \% 20,0 \%$} & \multicolumn{2}{|c|}{$75,0 \% 25,0 \%$} & \multicolumn{2}{|c|}{$72,5 \% 27,5 \%$} & \multicolumn{2}{|c|}{$70,0 \% 30,0 \%$} & \multicolumn{2}{|c|}{$70,0 \% 30,0 \%$} \\
\hline Masculino & \multicolumn{2}{|c|}{$69,6 \% 30,4 \%$} & \multicolumn{2}{|c|}{$71,4 \% 28,6 \%$} & \multicolumn{2}{|c|}{$80,4 \% 19,6 \%$} & \multicolumn{2}{|c|}{$75,0 \% 25,0 \%$} & \multicolumn{2}{|c|}{$66,1 \% 33,9 \%$} & \multicolumn{2}{|c|}{$71,4 \% 28,6 \%$} & \multicolumn{2}{|c|}{$71,4 \% 28,6 \%$} & \multicolumn{2}{|c|}{$69,6 \% 30,4 \%$} \\
\hline
\end{tabular}

Tabla 3. Impacto dela caries con respecto al desempeño diario

\begin{tabular}{lcc}
\hline & ENFERMO \\
\hline & $\mathbf{n}$ & $\%$ \\
CON IMPACTO & 54 & $67 \%$ \\
SIN IMPACTO & 27 & $33 \%$ \\
Total general & 81 & $100 \%$ \\
\hline
\end{tabular}

Tabla 4. Relación de la caries y su impacto en los desempeños diarios.

\section{DISCUSIÓN}

Se determina que los escolares atendidos corresponde al $42 \%$ al sexo femenino y $58 \%$ al masculino, este estudio tiene similitud con el estudio publicado por Rojas Venezuela $\mathrm{R}$, en la Comuna de Rio Hurtado en Chile, que presentó una prevalencia en el grupo masculino de $53 \%$ mientras que en el grupo femenino un $47 \% .{ }^{15} \mathrm{El}$ índice ceod en escolares de 6 años de edad dió a conocer la presencia de caries en niñas con el $39 \%$ y niños con el $46 \%$, dando como resultado que el $84 \%$ del total de la muestra presentó caries y el $16 \%$ correspondió a niños y niñas sanos; el cual tuvo semejanza con el estudio realizado por Fernández $\mathrm{C}$ at. Mendoza Argentina en el 2009, correspondiendo al sexo femenino al 84,1\% y el sexo masculino al $87,2 \% .{ }^{16}$ Cabe recalcar que el total de la muestra obtenida en este estudio no es cuantitativamente equivalente entre ambos sexos, teniendo una relación de 1.5: 1 entre hombres y mujeres.

Los impactos que afectaron los desempeños diarios fueron obtenidos mediante el test Child-OIDP reportando que el $67 \%$ presentó impacto por caries dental y el $33 \%$ no presentó impacto a pesar de la presencia de caries. Tal similitud presentó con el estudio realizado en Piura-Perú por Castillo López en el 2013 donde se observó que el 88,7 \% fue afectado por la caries dental en sus desempeños diarios y el $11,3 \%$ no lo reportó obteniendo un promedio $=4 \cdot 53 \cdot{ }^{17-19}$

Se demostró que los hombres presentan mayor impacto en los desempeños diarios con un $68 \%$ mientras que las mujeres un $58 \%$; el cual presentó similitud con el estudio realizado por Castillo López. ${ }^{20,21}$ en Piura en el 2013, siendo el $59 \%$ varones y el $41 \%$ mujeres; ambos estudios los varones presenta similitud con estudios realizados en España en el 2015 y Perú en el 2013, donde se observó que el desempeño con mayor impacto fue en el sexo masculino por "comer alimentos" con un 59\% y "hablar claramente" con un $44,3 \%$ en el sexo femenino, en cuanto al presente estudio dio como resultado que el $68 \%$ de los escolares de sexo masculino, el $16 \%$ presentó mayor impacto "comer alimentos" con un grado de severidad "mucho", mientras que el $58 \%$ de escolares de sexo femenino el $15 \%$ presentó mayor impacto en "contactarse con otras personas" con un grado de severidad "poco".22-24 El presenté estudio demostró que el sexo masculino tuvo mayor impacto que el sexo femenino en sus actividades diarias.

Este estudio nos permite tener un campo abierto para continuar proyectos de seguimiento en dicha población, con el fin de observar los factores causantes de enfermedad dental y el impacto que causa en sus actividades, para esto se vió importante los siguientes puntos: edad del escolar, instrucción y seguimiento de los padres o tutores hacia los escolares en cuanto a higiene bucal, el nivel socioeconómico y las diferencias por género de esta manera determinar los posibles elementos que agravan este problema de salud.

\section{CONCLUSIÓN}

El presente estudio concluyó que existe estrecha relación entre la caries dental y el impacto en los desempeños diarios de los escolares de 6 años en la parroquia Cañaribamba. Se determinó que la prevalencia de caries dental con respecto al sexo es mayor en el grupo masculino con relación al grupo femenino. También se demostró que el impacto en los desempeños diarios producto de la caries dental, es mayor en el sexo masculino sobre todo al momento de comer alimentos, en contraposición con el sexo femenino cuyo impacto se genera al momento de contactarse con otras personas.

Conflicto de intereses y financiamiento Los autores declaran no tener conflicto de intereses, haber cumplido con los requisitos de autoría y haber autofinanciado este artículo.

\section{Referencias}

1 Ramón R, Deroncelé M, Corona M, Estrada G, Quinzán A. Factores de riesgo de caries dental en escolares de 5 a 11 años. MEDISAN [Internet]. 2016 [citado 21 Nov 2016]; 
20(5): 604-610. Disponible en: Documento disponible

2 Ramírez B, Franco A, Ochoa E, Escobar G. Experiencia de caries en dentición primaria en niños de 5 años, Medellín, Colombia. Rev. Fac. Nac. Salud Pública [Internet]. 2015 [citado 21 Nov 2016]; 33(3): 345-352. Disponible en: Documento disponible

3 Díaz S, González F. Prevalencia de caries dental y facto- 15 res familiares en niños escolares de Cartagena de Indias, Colombia. Rev. salud pública [Internet]. 2010 [citado 21 Nov 2016]; 12 (5): 843-85. Disponible en: Documento disponible

4 Aguilar C, De la Fuente J, Shimada H, Acosta L. Instrumentos validados para medir la salud bucal en los niños. Salud y Ciencia [Internet]. 2014 [citado 21 Nov 2016]; 20: 846-851. Documento disponible

5 Castillo C, Evangelista A, Sánchez P. Impacto de la condiciones bucales sobre la calidad de vida en escolares de ámbito rural, Piura, Perú. Rev Estomatol Herediana [Internet]. 2014 [citado 21 Nov 2016]; 24(4): 220-228. Disponible en: Documento disponible

6 Rojas R, Camus M. Estudio Epidemiológico de las Caries Según Índice c.e.o.d y C.O.P.D. en Preescolares y Escolares de la Comuna de Río Hurtado, IV Región. Revista Dental de Chile [Internet]. 2001 [citado 21 Nov 2016]; 92 (1): 17-22. Documento disponible

7 Guerrero V, Godinez A, Melchor C, Rodriguez M, Quintero E. Epidemiología de caries dental y factores de riesgo asociados a la dentición primaria en preescolares. Revista ADM [Internet]. 2009 [citado 21 Nov 2016]; 65(3): 10-20. Documento disponible

8 Duque C, Mora I. La representación de la epidemiología de la caries en el mundo a través de mapas. Univ Odontol [Internet]. 2012 [citado 21 Nov 2016]; 31(66): 41-50. Documento disponible

9 Colimon K. Fundamentos de epidemiologia. Díaz de Santos S.A.; 1990.

10 Ojeda María Acosta N, Duarte E, Mendoza N, Meana María. Prevalencia de Caries Dental en Niños y Jóvenes de Zonas Rurales. Documento disponible

11 Cuartas J, Alvar A, Maya A, Cárdenas J, Arias M, Jaramillo A. Relación entre percepción de los padres sobre el tratamiento odontológico y sus hábitos de higiene oral, con la historia de caries dental en sus hijos, entre 3 y 5 años de edad. Rev. CES odontol [Internet]. 2002 [citado 21 Nov 2016]; 15(1): 13-18. Documento disponible

12 Condición del primer molar permanente en una población de escolares de la ciudad de Medellín 2012. Rev. CES odontol [Internet]. 2013 [citado 21 Nov 2016]; 26(1): 24 32. Documento disponible

13 Villena R, Pachas F, Sánchez Y, Carrasco M. Prevalencia 23 de caries de infancia temprana en niños menores de 6 años de edad, residentes en poblados urbano marginales de Lima Norte. Rev Estomatol Herediana [Internet]. 2011 [citado 21
Nov 2016]; 21(2): 79-86 Documento disponible

14 Vallejos A, Minaya M, Casanova J, Casanova A, Macias J, Vera S, et al. Gravedad de caries empleando un criterio del tamaño de la lesión y variables asociadas en una muestra de escolares mexicanos. Revista de la Universidad Industrial de Santander [Internet]. 2015 [citado 21 Nov 2016]; 47(3): 291-299. Documento disponible

5 Gálvez C. Prevalencia de caries dental en niños con dentición decidua usando índices ceo y el sistema internacional de detección y valoración de caries (Icdas II). Revista de Investigación de la Universidad Norbert Wiener [Internet]. 2013 [citado 21 Nov 2016]; 2: 117-127 Documento disponible

16 Fernandez C, Cambria S, Vuoto E, Borjas M, Giamportone V. Caries de la infancia temprana, demanda de atención odontológica y percepción parental de salud bucal en preescolares de riesgo social del Gran Mendoza. Facultad de odontología UN. Cuyo. [Internet]. 2010 [citado 21 Nov 2016]; 4(2): 29-37 Documento disponible

17 Romero Y, Carrillo D, Espinoza N, Díaz N. Perfil epidemiológico en salud bucal de la población escolarizada del municipio campo Elías del estado Mérida. Acta Bioclínica [Internet]. 2016 [citado 21 Nov 2016]; 6(11): 3-24. Documento disponible

18 Torres H. Estudio epidemiológico sobre caries dental y necesidades de tratamiento en escolares de 3 a 5 años de edad de Huacho, Perú. Revista Salud, Sexualidad y Sociedad [Internet]. 2010 [citado 21 Nov 2016]; 3(1). Documento disponible

19 Villavicencio E, Cuenca K, Vélez E, Sayago J, Cabrera A. Pasos para la planificación de un estudio clínico, 2016. Odontología activa. Vol $1 \mathrm{~N} 1$ Universidad Católica de Cuenca ISSN: 2477-8915..

20 Villavicencio E. El tamaño muestral en tesis de postgrado. ¿Cuántas personas debo encuestar?, 2011.Odontología activa. Vol 1 N 1 Universidad Católica de Cuenca ISSN: 24778915.

21 Villalobos J, Medina C, Molina N, Vallejos A, Pontigo A, Espinoza J. Caries dental en escolares de 6 a 12 años de edad en Navolato, Sinaloa, México: experiencia, prevalencia, gravedad y necesidades de tratamiento. Biomedia [Internet]. 2006 [citado 21 Nov 2016]; 26: 224-233. Documento disponible

22 Rodríguez M, Mursulí M, Pérez L, Martínez M. Estado de salud del primer molar permanente en niños de 6-11 años. Sancti Spíritus. 2011. Gaceta Médica Espirituana [Internet] 2013 [citado 21 Nov 2016]; 15(1). Documento disponible

3 Mendes, D. Caricote, N. Prevalencia de Caries Dental en Escolares de 6 a 12 años de edad del Municipio Antolín del Campo, Estado Nueva Esparta, Venezuela (2002-2003). evista Latinoamericana de Ortodoncia y 
Odontopediatria [Internet].2003 [citado 21 Nov 2016].

Recibido: 13 de enero de 2017.

Documento disponible

Aceptado: 17 de marzo de 2017.

24 Montero J. Salud oral relacionada con la calidad de vida en escolares de 6 a 12 años de edad en España. Revista internacional de odontología pediátrica [Internet]. 2015 [citado 21

Nov 2016]; Documento disponible 
